\title{
Application of Lactoferrin and $\alpha 1$-Antitrypsin in Gingival Retention Fluid to Diagnosis of Periodontal Disease
}

\author{
Ryosuke Koshi, ${ }_{1}^{1}$ Kazuhiko Kotani $\left(\mathbb{D},{ }^{2}\right.$ Mariko Ohtsu, ${ }^{3}$ Naoto Yoshinuma, ${ }^{1}$ \\ and Naoyuki Sugano $\mathbb{1}^{1}$ \\ ${ }^{1}$ Department of Periodontology, Nihon University School of Dentistry, Tokyo, Japan \\ ${ }^{2}$ Division of Community and Family Medicine, Jichi Medical University, Tochigi, Japan \\ ${ }^{3}$ Department of Pathology, Nihon University School of Dentistry, Tokyo, Japan
}

Correspondence should be addressed to Naoyuki Sugano; sugano.naoyuki@nihon-u.ac.jp

Received 10 August 2018; Revised 26 September 2018; Accepted 8 October 2018; Published 7 November 2018

Guest Editor: Napoleon Waszkiewicz

Copyright ( 2018 Ryosuke Koshi et al. This is an open access article distributed under the Creative Commons Attribution License, which permits unrestricted use, distribution, and reproduction in any medium, provided the original work is properly cited.

\begin{abstract}
Objectives. Periodontal disease is prevalent and has an inflammation associated with not only oral but also systemic pathologies. The diagnosis by biomarkers is required for clinical practice on periodontal disease. The lactoferrin and $\alpha 1$-antitrypsin were both inflammation-related molecules. The present study investigated the relationship between the periodontal status and the two biomarkers in gingival retention fluid (GRF). Patients and Methods. In 63 subjects with periodontitis, the GRF was sampled from maxillary anterior gingiva using a microbrush for 30 seconds. The lactoferrin and $\alpha 1$-antitrypsin levels in GRF were measured by an enzyme-link solvent immunoassay. Periodontal status was evaluated by probing pocket depth (PD) and bleeding on probing (BOP). Results. There was a higher level of these biomarkers in saliva (median (ng/mL), lactoferrin: 3611.9, $\alpha 1$-antitrypsin: 4573.3) than in GRF (lactoferrin: 61.0, $\alpha 1$-antitrypsin: 54.7). There was a mild-to-moderate but significantly positive correlation in lactoferrin or $\alpha 1$-antitrypsin between GRF and saliva. There was a positively mild-to-moderate accuracy (area under the curve: $0.60-0.81$ ) of lactoferrin or $\alpha 1$-antitrypsin in GRF or in saliva to distinguish the severity of periodontal status. The cutoff level ( $\mathrm{ng} / \mathrm{mL}$ ) of lactoferrin in GRF for detecting $\geq 30 \%$ of $\mathrm{PD} \geq 4 \mathrm{~mm}$ (moderate periodontitis) was 68.6 and for detecting $\geq 20 \%$ of BOP (clinically active periodontitis) was 61.2 . The cutoff level $(\mathrm{ng} / \mathrm{mL})$ of $\alpha 1$-antitrypsin in GRF for detecting $\geq 30 \%$ of $\mathrm{PD} \geq 4 \mathrm{~mm}$ was 54.5 and for detecting $\geq 20 \%$ of BOP was 35.3. Conclusions. The data can promote an application of the measurements of lactoferrin and $\alpha 1$-antitrypsin in GRF to clinical practice on periodontal disease.
\end{abstract}

\section{Introduction}

Periodontal disease is prevalent (up to 90\%) across countries [1]. This disease, often caused by bacterial invasion, promotes the attachment of connective tissue and the protection of bone around the teeth at the early step of disease, but its subsequent formation of inflammation contributes to the destruction of periodontal tissues $[2,3]$. It is thus a chronic inflammatory disorder, which induces not only locally oral but also systemic bodily pathologies $[4,5]$. Nowadays, the management of this disease is widely recognized to be crucial.

The diagnosis of periodontal disease has so far relied on human hands to measure the periodontal tissue [6, 7]. Easy and objective measurements using biomarkers are indeed required. Recently, several biomarkers, such as C-reactive protein or bacteria-related DNA/enzyme in saliva and gingival crevicular fluid, have been arisen as the candidates; however, the use of such biomarkers for periodontal disease has not yet to be established [8-10].

The lactoferrin is primarily originated in neutrophils, which response to an acute inflammation [11, 12]. The lactoferrin is enhanced in an anti-inflammatory action through the binding to the lipid A portion of lipopolysaccharide of bacteria [11]. Therefore, lactoferrin is considered an inflammation-related molecule $[11,12]$. Also, protease inhibitors are anti-inflammatory reactants, and the $\alpha 1$-antitrypsin (a protease inhibitor) derived from serum (i.e., throughout exudate and bleeding) is enhanced by inflammatory cytokines and endotoxins $[13,14]$. Therefore, the $\alpha 1$-antitrypsin is also considered an inflammation-related molecule $[13,14]$. 
Limited studies (with the different assays) have previously investigated the lactoferrin level or $\alpha 1$-antitrypsin level in saliva and in gingival crevicular fluid for periodontal disease and gingival disease [15-19]. An increase of these molecules is suggested to be the potential biomarkers for such diseases [15-19]. Accordingly, the lactoferrin and $\alpha 1$-antitrypsin levels using oral materials with an enzyme immunoassay have been measured; the clinical ability of the measurements remains to be determined in daily practice on periodontal disease [20]. The present study investigated the relationship between the periodontal status and these two biomarkers in gingival retention fluid (GRF; a mixture of saliva and gingival crevicular fluid) in comparison to that in saliva (a classical material of this field).

\section{Patients and Methods}

2.1. Patients and Sample Measurements. A total of 63 subjects, who visited to clinics for checking the periodontal status, were consecutively enrolled into the current study. Subjects with apparent inflammatory diseases (e.g., respiratory or bowel infection) were excluded. The study was approved by the ethics committee of the Nihon University School of Dentistry (no. EP13D15). Written informed consent was obtained from all participants before their inclusion into the study.

The clinical criteria for periodontal disease (especially periodontitis) were judged from the standard measurements of clinical probing depth. The sampling for lactoferrin and $\alpha 1$-antitrypsin in GRF or saliva was performed at the same time. The GRF was collected from maxillary anterior gingiva using a microbrush for 30 seconds before eating any foods [20]. Parafin wax-stimulated whole saliva was collected before clinical examination. Samples were stored in the specific tubes that were applied to measure the lactoferrin and $\alpha 1$-antitrypsin levels by an enzymelink solvent immunoassay using a monoclonal antibody of lactoferrin and $\alpha 1$-antitrypsin (which was developed by Ikagaku Co., Ltd. (Kyoto, Japan)) [20]. The coefficient of variation regarding assays was 3.7\% in lactoferrin and $2.6 \%$ in $\alpha 1$-antitrypsin, respectively.

Periodontal disease was evaluated by probing pocket depth (PD) [21]. The PD does not always reflect current periodontal inflammation. The assessment of bleeding on probing (BOP) may be reflective to an active inflammation of periodontal tissue [21]. Specifically, the percentage of sites with a $P D \geq 4 \mathrm{~mm}$ was calculated, and clinically moderate periodontitis was defined as $\geq 30 \%$ [22]. Immediately thereafter, the BOP was recorded as present or absent at six sites per tooth. The percentage of sites with BOP was calculated, and clinically active periodontitis was defined at BOP $\geq 20 \%$ [23].

2.2. Statistical Analysis. The data are presented as the mean \pm standard deviation (for variables with normal distributions), the median and interquartile range (for variables with skewed distributions), or subject number. The difference between the two groups was analyzed by the Student $t$-test. A simple correlation test (Pearson's correlation test) was used to analyze the correlation between variables. A multiple
TABLE 1: Clinical data of the study subjects.

\begin{tabular}{lc}
\hline Variable & Levels \\
\hline Age, years & $48 \pm 16$ \\
Gender (men/women), number & $33 / 30$ \\
Prevalence of PD $\geq 4 \mathrm{~mm}(\%)$ & $10.5(1.1-30.9)$ \\
Subjects with $\geq 30 \%$ of PD $\geq 4 \mathrm{~mm}$, & $16(25 \%)$ \\
number (\%) & $19.8(10.5-45.8)$ \\
BOP (\%) & $31(49 \%)$ \\
Subjects with $\geq 20 \%$ of BOP, & 61.0 \\
number (\%) & $(33.8-117.8)^{\mathrm{a} * *}$ \\
Lactoferrin in GRF (ng/mL) & 3611.9 \\
& $(2789.1-7751.2)^{\mathrm{a} * *}$ \\
Lactoferrin in saliva (ng/mL) & 54.7 \\
$\alpha 1$-antitrypsin in GRF (ng/mL) & $(23.2-212.5)^{\mathrm{b} * *}$ \\
$\alpha 1$-antitrypsin in saliva (ng/mL) & 4573.3 \\
& $(2122.0-10834.1)^{\mathrm{b} * *}$ \\
\hline
\end{tabular}

PD: probing pocket depth, BOP: bleeding on probing, GRF: gingival retention fluid. The data are presented as the mean \pm standard deviation, median (interquartile range), or patient number (\%). Significance level (gingival sulcus vs. saliva; ${ }^{a}$ lactoferrin, ${ }^{\mathrm{b}} \alpha 1$-antitrypsin): ${ }^{* *} P<0.01$.

regression analysis was also used to analyze the correlation between variables with adjustment for basic confounders such as age and gender. A receiver operating characteristic (ROC) curve analysis was used to identify cutoff levels of lactoferrin and $\alpha 1$-antitrypsin for detecting the outcome. The values of variables with skewed distributions were log transformed in these analyses. A statistical significance ( $P$ value) was set as $<0.05$.

\section{Results}

Table 1 shows the clinical data of study subjects. The lactoferrin level was higher in saliva than in GRF. The $\alpha 1$-antitrypsin level was also higher in saliva than in GRF. The lactoferrin level in GRF was insignificantly different from the $\alpha 1$-antitrypsin level in GRF $(P>0.05)$. The lactoferrin level in saliva was also insignificantly different from the $\alpha 1$-antitrypsin level in saliva $(P>0.05)$.

Table 2 shows the simple correlation of lactoferrin in GRF or saliva with other variables. There was a significantly positive correlation of lactoferrin between GRF and saliva ( $r=0.43, P<0.01)$, and the correlation remained to show the same trend after adjusting age and gender $(\beta=0.42, P$ $<0.01)$. There was a significantly positive correlation between the prevalence of $\mathrm{PD} \geq 4 \mathrm{~mm}$ and lactoferrin in GRF or saliva. These correlations showed the similar trend after adjusting age and gender (GRF: $\beta=0.29, P=0.03$, saliva: $\beta=0.42, P<0.01)$. Also, there was a significantly positive correlation between BOP and lactoferrin in GRF or in saliva. These correlations showed the same trend after adjusting age and gender (GRF: $\beta=0.23, P=0.08$, saliva: $\beta=0.41, P<0.01)$. Finally, there was a significantly positive correlation between lactoferrin and $\alpha 1$-antitrypsin, and the correlation was relatively high between lactoferrin 
TABLE 2: Correlation of lactoferrin in GRF or saliva with variables.

\begin{tabular}{lcc}
\hline Variable & GRF & Saliva \\
\hline Age & $-0.03(0.84)$ & $0.11(0.38)$ \\
Male gender & $0.20(0.12)$ & $0.11(0.38)$ \\
Prevalence of PD $\geq 4 \mathrm{~mm}$ & $0.29\left(0.02^{*}\right)$ & $0.43\left(<0.01^{* *}\right)$ \\
BOP & $0.25\left(0.047^{*}\right)$ & $0.42\left(<0.01^{* *}\right)$ \\
Lactoferrin in saliva & $0.43\left(<0.01^{* *}\right)$ & - \\
$\alpha 1$-antitrypsin in GRF & $0.61\left(<0.01^{* *}\right)$ & $0.39\left(<0.01^{* *}\right)$ \\
$\alpha 1$-antitrypsin in saliva & $0.44\left(<0.01^{* *}\right)$ & $0.69\left(<0.01^{* *}\right)$ \\
\hline
\end{tabular}

PD: probing pocket depth, BOP: bleeding on probing, GRF: gingival retention fluid. The data are presented as correlation coefficient $r$ ( $p$-value) by simple correlation test (Pearson test). Significance level: ${ }^{*} P<0.05,{ }^{* *} P<0.01$.

and $\alpha 1$-antitrypsin in GRF or between lactoferrin and $\alpha 1$ antitrypsin in saliva.

Table 3 shows the simple correlation of $\alpha 1$-antitrypsin in GRF or saliva with other variables. There was a significantly positive correlation of $\alpha 1$-antitrypsin between GRF and saliva $(r=0.53, P<0.01)$, and the correlation remained to show the same trend after adjusting age and gender $(\beta=0.52, P<0.01)$. There was a significantly positive correlation between the prevalence of $\mathrm{PD} \geq 4 \mathrm{~mm}$ and $\alpha 1$-antitryp$\sin$ in GRF or in saliva. These correlations showed the same trend after adjusting age and gender (GRF: $\beta=0.33, P=$ 0.02 , saliva: $\beta=0.53, P<0.01$ ). Also, there was a significantly positive correlation between BOP and $\alpha 1$-antitrypsin in GRF or in saliva. These correlations showed the same trend after adjusting age and gender (GRF: $\beta=0.39, P<0.01$, saliva: $\beta=0.57, P<0.01$ ).

Table 4 and Figure 1 show the ROC curve analysis of lactoferrin in GRF or in saliva. The area under the curve (AUC) indicated a significantly moderate accuracy for $\geq 30 \%$ of $\mathrm{PD} \geq 4 \mathrm{~mm}$ (moderate periodontitis), and the cutoff value (ng/mL) for detecting $\geq 30 \%$ of $\mathrm{PD} \geq 4 \mathrm{~mm}$ was 68.6 in GRF and 7585.8 in saliva. The AUC of lactoferrin in saliva indicated a significantly moderate accuracy, while the AUC of lactoferrin in GRF indicated a relatively low accuracy for $\geq 20 \%$ of BOP (clinically active periodontitis). The cutoff value (ng/mL) for detecting $\geq 20 \%$ of BOP was 61.2 in GRF and 3715.4 in saliva.

Table 5 and Figure 2 show the ROC curve analysis of $\alpha 1$ antitrypsin in GRF or in saliva. Overall, the AUC of $\alpha 1$-antitrypsin appeared to be high relative to that of lactoferrin, while the accuracies of AUC of $\alpha 1$-antitrypsin were also at moderate levels for outcomes. The AUC indicated a significantly moderate accuracy for $\geq 30 \%$ of $P D \geq 4 \mathrm{~mm}$, and the cutoff value (ng/mL) for detecting $\geq 30 \%$ of $P D \geq 4 \mathrm{~mm}$ was 54.5 in GRF and 8871.6 in saliva. The AUC indicated a significantly moderate accuracy for $\geq 20 \%$ of BOP, and the cutoff value (ng/mL) for detecting $\geq 20 \%$ of BOP was 35.3 in GRF and 4265.8 in saliva.

\section{Discussion}

The present study is the first to investigate clinically the relationships among the periodontal status, lactoferrin, and $\alpha 1$-antitrypsin in GRF, in a comparative manner of
TABLE 3: Correlation of $\alpha 1$-antitrypsin in GRF or in saliva with variables.

\begin{tabular}{lcc}
\hline Variable & GRF & Saliva \\
\hline Age & $0.16(0.22)$ & $0.04(0.73)$ \\
Male gender & $0.12(0.36)$ & $-0.03(0.80)$ \\
Prevalence of PD $\geq 4 \mathrm{~mm}$ & $0.36\left(<0.01^{* *}\right)$ & $0.46\left(<0.01^{* *}\right)$ \\
BOP & $0.42\left(<0.01^{* *}\right)$ & $0.50\left(<0.01^{* *}\right)$ \\
$\alpha 1$-antitrypsin in saliva & $0.53\left(<0.01^{* *}\right)$ & - \\
\hline
\end{tabular}

PD: probing pocket depth, BOP: bleeding on probing, GRF: gingival retention fluid. The data are presented as correlation coefficient $r$ ( $p$-value) by simple correlation test (Pearson test). Significance level: ${ }^{*} P<0.05,{ }^{* *} P<0.01$.

their relationships in saliva, using an enzyme immunoassay. Saliva is a classical material of this research field, while the use of GRF is reasonable as it is close to periodontal disease. The conventional GCF (gingival crevicular fluid) is collected from the gingival sulcus with one tooth using a paper point. The sampling method using the microbrush used in this study is different from the original GCF. Therefore, we defined newly as GRF (gingival retention fluid). Saliva reflects the entire oral cavity, whereas GCF is considered to reflect the gingival condition of each tooth. However, sampling of GCF is time-consuming and requires certain skills. In contrast, GRF reflects a wider range of gingival conditions, and simple sampling methods can be applied to mass screening. The results of the present study would be valuable to offer the insight in an application of measurements of lactoferrin and $\alpha 1$-antitrypsin with the use of GRF to clinical practice on periodontal disease.

The first finding of this study is a moderate correlation between lactoferrin and $\alpha 1$-antitrypsin in GRF or in saliva in this population. The two biomarkers are both inflammation-related molecules [11-14], and their increase in periodontal and gingival disease has been previously reported [15-19]. Therefore, the correlation appears to be natural, even though these can have a different pathophysiological origin [11-14]. The overlapping and/or independent application of these biomarkers to clinics is a next issue.

The second finding is a mild-to-moderate correlation in lactoferrin or $\alpha 1$-antitrypsin between GRF and saliva, while a higher level of the biomarkers in saliva than in GRF. This appeared to be simply reflective to the difference in the amount of sampled materials.

The third finding (from the results of correlation and ROC curve analyses) is a positively mild-to-moderate accuracy of the lactoferrin level with the severity of periodontal status, as well as the $\alpha 1$-antitrypsin level with the severity of periodontal status, in GRF or saliva. These may mean that the measurements of lactoferrin and $\alpha 1$-antitrypsin in GRF are available for the diagnosis of periodontal disease. The present study newly provided the cutoff levels on the severity of periodontal status in lactoferrin and $\alpha 1$-antitrypsin. Their diagnostic abilities did not necessarily seem to be very high but were moderate, indicating that it could be useful to apply the assays to clinics as a supplemental tool. Under this situation, the lactoferrin in GRF weakly distinguished the 
TABLE 4: ROC curve analysis of lactoferrin in GRF or saliva.

\begin{tabular}{|c|c|c|c|c|c|c|c|}
\hline Outcomes & AUC (95\% CI) & $P$ value & Cutoff (ng/mL) & Sensitivity & Specificity & PLR & NLR \\
\hline \multicolumn{8}{|l|}{ For $\geq 30 \%$ of $\mathrm{PD} \geq 4 \mathrm{~mm}$} \\
\hline Lactoferrin in GRF & $0.76(0.60-0.92)$ & $<0.01^{* *}$ & 68.6 & 0.81 & 0.72 & 2.9 & 0.3 \\
\hline Lactoferrin in saliva & $0.67(0.50-0.84)$ & $0.04^{*}$ & 7585.8 & 0.50 & 0.83 & 2.9 & 0.3 \\
\hline \multicolumn{8}{|l|}{ For $\geq 20 \%$ of BOP } \\
\hline Lactoferrin in GRF & $0.60(0.46-0.75)$ & 0.16 & 61.2 & 0.55 & 0.56 & 1.3 & 0.8 \\
\hline Lactoferrin in saliva & $0.70(0.57-0.83)$ & $<0.01^{* *}$ & 3715.4 & 0.61 & 0.72 & 3.6 & 0.5 \\
\hline
\end{tabular}

ROC: receiver operating characteristic, PD: probing pocket depth, BOP: bleeding on probing, GRF: gingival retention fluid, AUC: area under the curve, CI: confidence interval, PLR: positive likelihood ratio, NLR: negative likelihood ratio. Significance level: ${ }^{*} P<0.05,{ }^{* *} P<0.01$.

For $\geq 30 \%$ of $\mathrm{PD} \geq 4 \mathrm{~mm}$
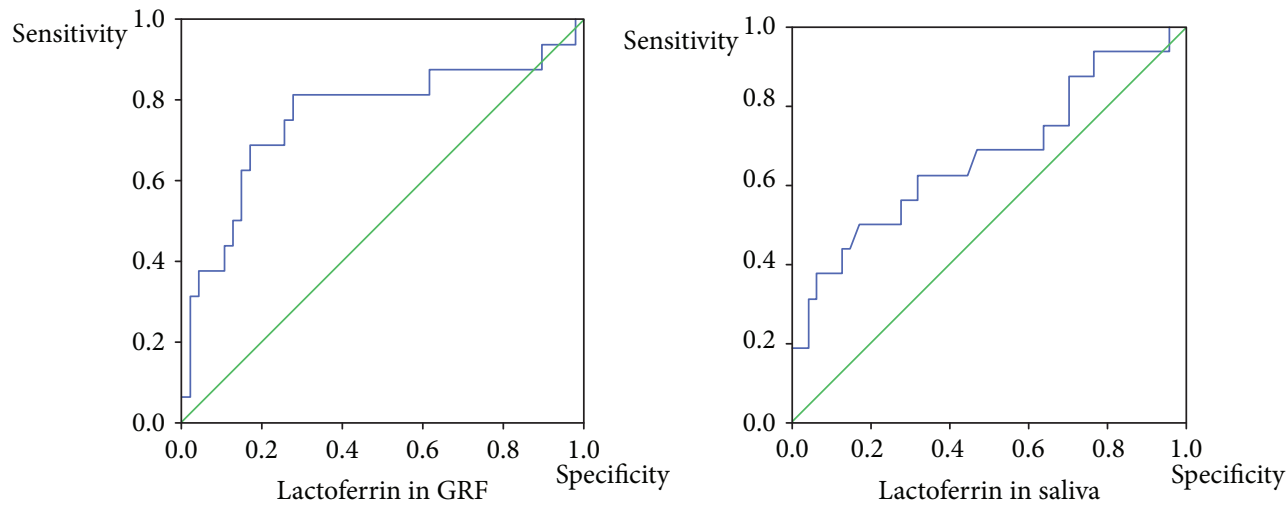

For $\geq 20 \%$ of BOP
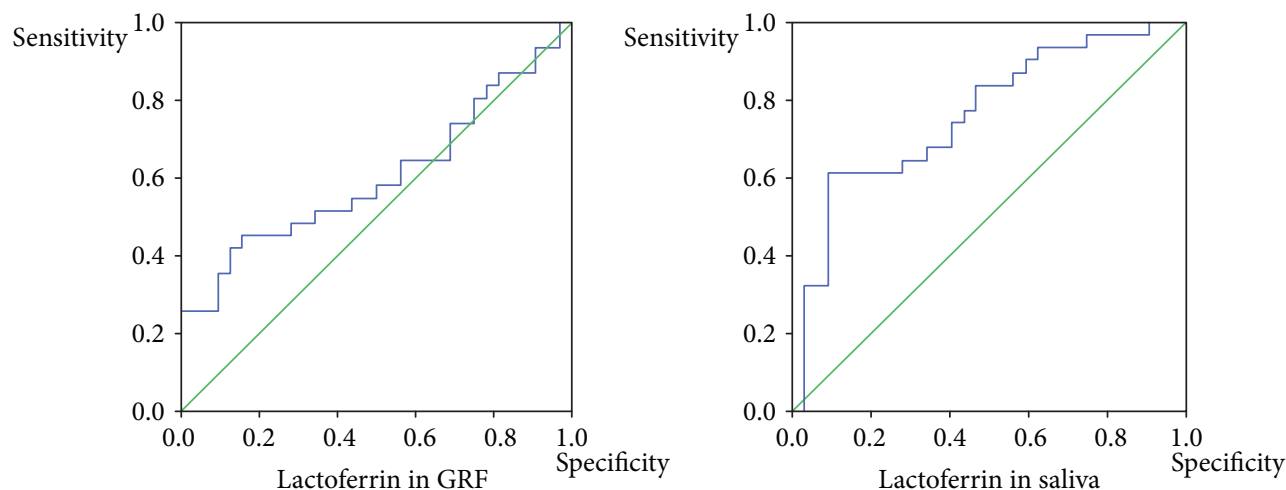

FIGURE 1: ROC curve analysis of lactoferrin in GRF or in saliva.

TABLE 5: ROC curve analysis of $\alpha 1$-antitrypsin in GRF or in saliva.

\begin{tabular}{|c|c|c|c|c|c|c|c|}
\hline Outcomes & AUC (95\% CI) & $P$ value & Cutoff (ng/mL) & Sensitivity & Specificity & PLR & NLR \\
\hline \multicolumn{8}{|l|}{ For $\geq 30 \%$ of $\mathrm{PD} \geq 4 \mathrm{~mm}$} \\
\hline$\alpha 1$-antitrypsin in GRF & $0.76(0.62-0.90)$ & $<0.01^{* *}$ & 54.5 & 0.81 & 0.60 & 2.0 & 0.5 \\
\hline$\alpha 1$-antitrypsin in saliva & $0.77(0.65-0.90)$ & $<0.01^{* *}$ & 8871.6 & 0.69 & 0.83 & 4.0 & 0.2 \\
\hline \multicolumn{8}{|l|}{ For $\geq 20 \%$ of $\mathrm{BOP}$} \\
\hline$\alpha 1$-antitrypsin in GRF & $0.76(0.64-0.88)$ & $<0.01^{* *}$ & 35.3 & 0.84 & 0.53 & 1.8 & 0.6 \\
\hline$\alpha 1$-antitrypsin in saliva & $0.81(0.70-0.92)$ & $<0.01^{* *}$ & 4265.8 & 0.74 & 0.63 & 2.0 & 0.5 \\
\hline
\end{tabular}

ROC: receiver operating characteristic, PD: probing pocket depth, BOP: bleeding on probing, GRF: gingival retention fluid, AUC: area under the curve, CI: confidence interval, PLR: positive likelihood ratio, NLR: negative likelihood ratio. Significance level: ${ }^{*} P<0.05,{ }^{* *} P<0.01$. 


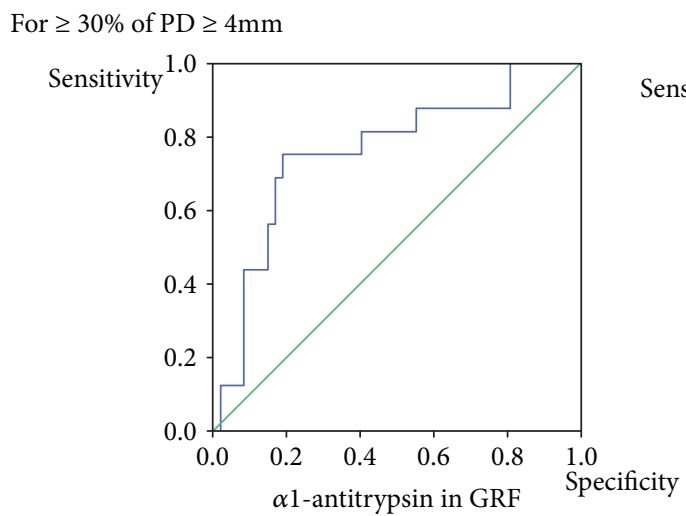

For $\geq 20 \%$ of BOP
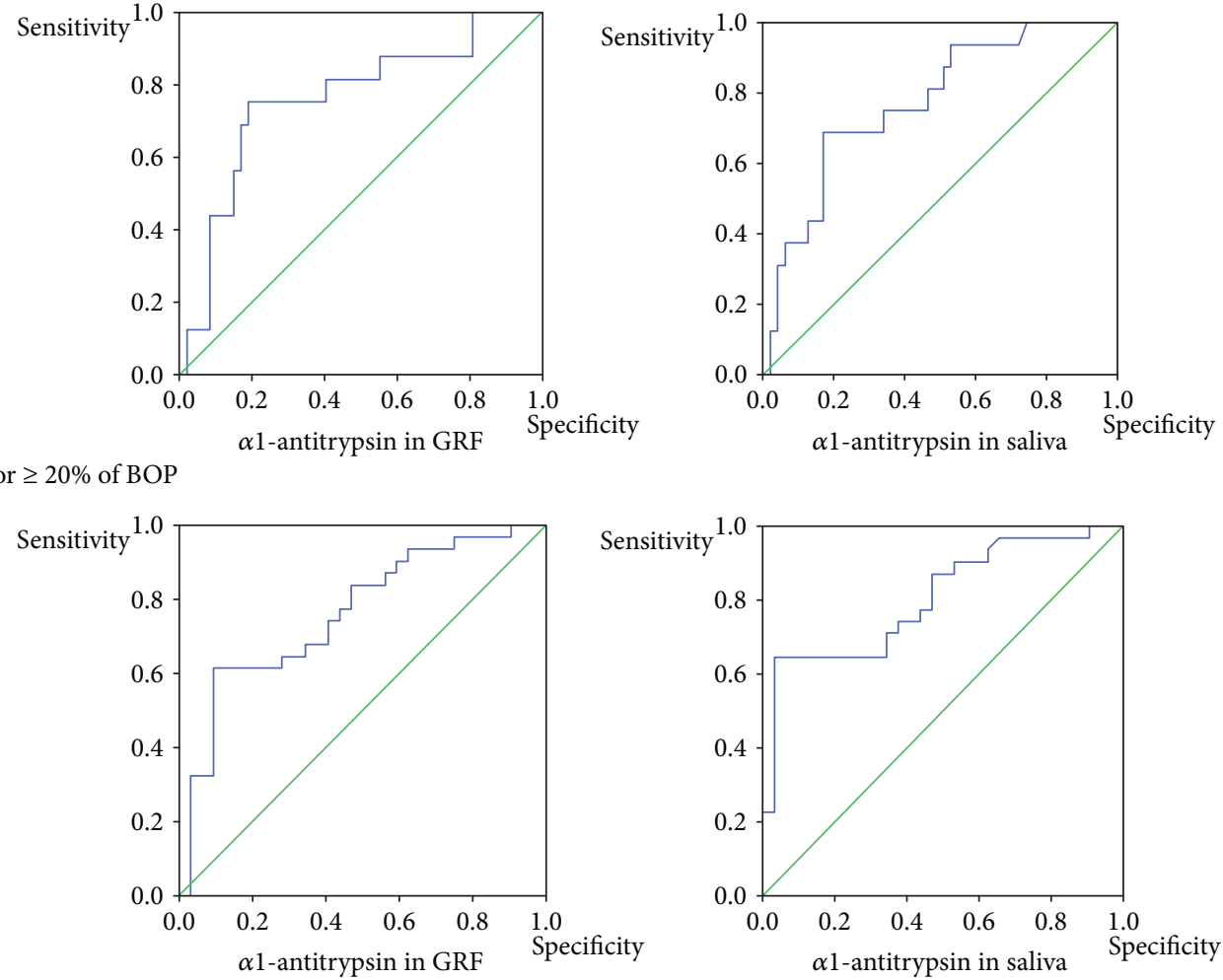

FIGURE 2: ROC curve analysis of $\alpha 1$-antitrypsin in GRF or in saliva.

severities by BOP. As the BOP is an indicator of active inflammation with exudate and bleeding, the $\alpha 1$-antitrypsin (a molecule derived from serum) can distinguish the severities by BOP relative to the lactoferrin, especially in case of the use of GRF. Whether the use of $\alpha 1$-antitrypsin is superior to that of lactoferrin in GRF in a specific condition like active inflammation merits a further confirmation. We are now investigating the change in the measurement value due to the improvement of clinical symptoms after treatment.

There was a limitation to the present study. The subject numbers studied were relatively small. The inflammatory molecules in blood and/or the additional inflammatory molecules in GRF were not measured. The microbrush to collect samples was manually operated, and its operation might not completely be standardized.

\section{Conclusions}

The present study demonstrated that the lactoferrin and $\alpha 1$ antitrypsin in GRF were positively related to the severity of periodontal status. The measurements of these biomarkers can be applied to clinical practice on periodontal disease, while more multifaced studies are warranted.

\section{Data Availability}

The authors may make data available on request through the authors themselves. In this case, they should name who should be contacted to request the data and provide appropriate contact details. The provision of data can be needed to be reviewed in the institutional ethics committee.

\section{Ethical Approval}

Ethical approval was obtained from the ethics committee of Nihon University School of Dentistry (no. EP13D15).

\section{Conflicts of Interest}

The authors declare that they have no conflicts of interest.

\section{Authors' Contributions}

RK and NS conceived and designed the study. MO and NY collected and compiled the data. NS wrote the first draft of the paper. KK interpreted the results and reviewed the paper.

\section{Acknowledgments}

This work was supported by a grant from the Dental Research Center, Nihon University School of Dentistry (2017; NS).

\section{References}

[1] B. L. Pihlstrom, B. S. Michalowicz, and N. W. Johnson, "Periodontal diseases," The Lancet, vol. 366, no. 9499, pp. 1809-1820, 2005. 
[2] H. Birkedal-Hansen, "Role of cytokines and inflammatory mediators in tissue destruction," Journal of Periodontal Research, vol. 28, no. 6, pp. 500-510, 1993.

[3] K. S. Kornman, R. C. Page, and M. S. Tonetti, "The host response to the microbial challenge in periodontitis: assembling the players," Periodontology 2000, vol. 14, no. 1, pp. 33-53, 1997.

[4] G. J. Linden, A. Lyons, and F. A. Scannapieco, "Periodontal systemic associations: review of the evidence," Journal of Periodontology, vol. 84, no. 4-s, pp. S8-19, 2013.

[5] K. Watanabe and Y. D. Cho, "Periodontal disease and metabolic syndrome: a qualitative critical review of their association," Archives of Oral Biology, vol. 59, no. 8, pp. 855-870, 2014.

[6] N. P. Lang, R. Adler, A. Joss, and S. Nyman, "Absence of bleeding on probing. An indicator of periodontal stability," Journal of Clinical Periodontology, vol. 17, no. 10, pp. 714-721, 1990.

[7] J. M. Goodson, "Diagnosis of periodontitis by physical measurement: interpretation from episodic disease hypothesis," Journal of Periodontology, vol. 63, no. 4s, pp. 373-382, 1992.

[8] N. Buduneli and D. F. Kinane, "Host-derived diagnostic markers related to soft tissue destruction and bone degradation in periodontitis," Journal of Clinical Periodontology, vol. 38, no. 11, pp. 85-105, 2011.

[9] J. J. Taylor and P. M. Preshaw, "Gingival crevicular fluid and saliva," Periodontology 2000, vol. 70, no. 1, pp. 7-10, 2016.

[10] S. P. Barros, R. Williams, S. Offenbacher, and T. Morelli, "Gingival crevicular fluid as a source of biomarkers for periodontitis," Periodontology 2000, vol. 70, no. 1, pp. 53-64, 2016.

[11] B. J. Appelmelk, Y. Q. An, M. Geerts et al., "Lactoferrin is a lipid A-binding protein," Infection and Immunity, vol. 62, no. 6, pp. 2628-2632, 1994.

[12] G. Embery and R. Waddington, "Gingival crevicular fluid: biomarkers of periodontal tissue activity," Advances in Dental Research, vol. 8, no. 2, pp. 329-336, 1994.

[13] E. Adonogianaki, J. Mooney, and D. F. Kinane, "Detection of stable and active periodontitis sites by clinical assessment and gingival crevicular acute-phase protein levels," Journal of Periodontal Research, vol. 31, no. 2, pp. 135-143, 1996.

[14] S. M. Janciauskiene, R. Bals, R. Koczulla, C. Vogelmeier, T. Köhnlein, and T. Welte, "The discovery of a1-antitrypsin and its role in health and disease," Respiratory Medicine, vol. 105, no. 8, pp. 1129-1139, 2011.

[15] E. Adonogianaki, N. A. Moughal, and D. R. Kinane, "Lactoferrin in the gingival crevice as a marker of polymorphonuclear leucocytes in periodontal diseases," Journal of Clinical Periodontology, vol. 20, no. 1, pp. 26-31, 1993.

[16] E. Adonogianaki, N. A. Moughal, J. Mooney, D. R. Stirrups, and D. F. Kinane, "Acute-phase proteins in gingival crevicular fluid during experimentally induced gingivitis," Journal of Periodontal Research, vol. 29, no. 3, pp. 196-202, 1994.

[17] H. Jentsch, Y. Sievert, and R. Göcke, "Lactoferrin and other markers from gingival crevicular fluid and saliva before and after periodontal treatment," Journal of Clinical Periodontology, vol. 31, no. 7, pp. 511-514, 2004.

[18] P. Glimvall, C. Wickström, and H. Jansson, "Elevated levels of salivary lactoferrin, a marker for chronic periodontitis," Journal of Periodontal Research, vol. 47, no. 5, pp. 655-660, 2012.

[19] S. W. Cox, E. M. Rodriguez-Gonzalez, V. Booth, and B. M. Eley, "Secretory leukocyte protease inhibitor and its potential interactions with elastase and cathepsin B in gingival crevicular fluid and saliva from patients with chronic periodontitis," Journal of Periodontal Research, vol. 41, no. 5, pp. 477-485, 2006.

[20] S. Hayashi, H. Yamada, M. Fukui, H. O. Ito, and M. Sata, "Correlation between arteriosclerosis and periodontal condition assessed by lactoferrin and a1-antitrypsin levels in gingival crevicular fluid," International Heart Journal, vol. 56, no. 6, pp. 639-643, 2015.

[21] E. S. Chaves, R. C. Wood, A. A. Jones, D. A. Newbold, M. A. Manwell, and K. S. Kornman, "Relationship of "bleeding on probing" and "gingival index bleeding" as clinical parameters of gingival inflammation," Journal of Clinical Periodontology, vol. 20, no. 2, pp. 139-143, 1993.

[22] R. C. Page and P. I. Eke, "Case definitions for use in population-based surveillance of periodontitis," Journal of Periodontology, vol. 78, no. 7s, pp. 1387-1399, 2007.

[23] A. Joss, R. Adler, and N. P. Lang, "Bleeding on probing. A parameter for monitoring periodontal conditions in clinical practice," Journal of Clinical Periodontology, vol. 21, no. 6, pp. 402-408, 1994. 


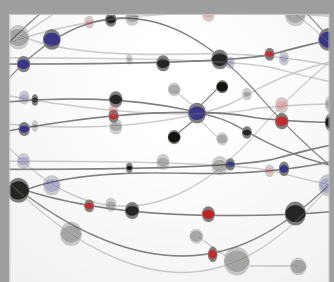

The Scientific World Journal
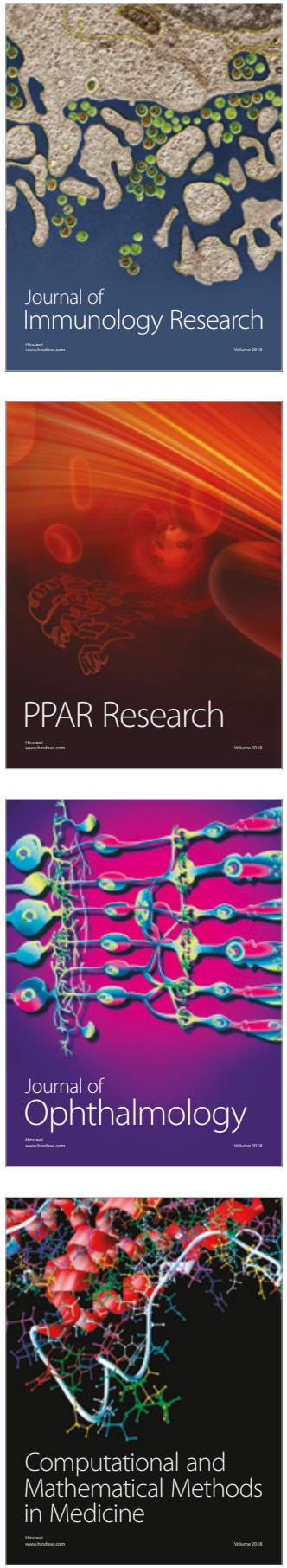

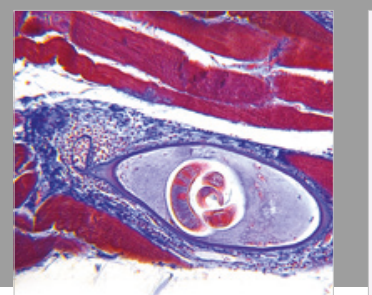

Gastroenterology Research and Practice

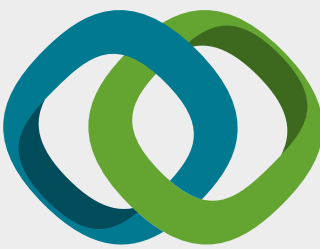

\section{Hindawi}

Submit your manuscripts at

www.hindawi.com
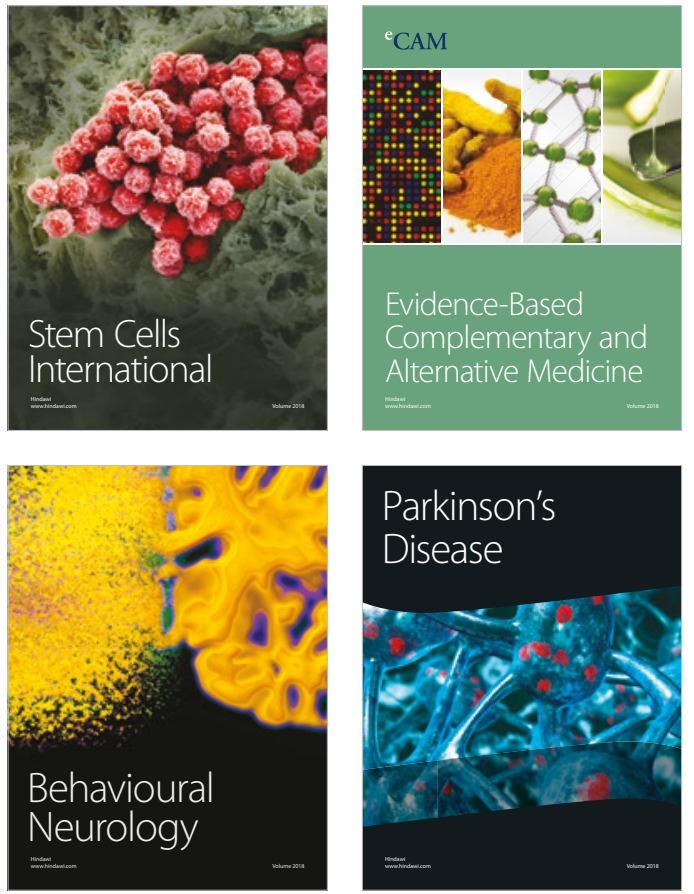

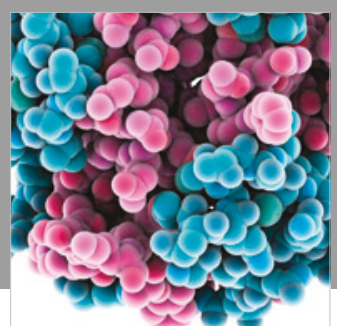

ournal of

Diabetes Research

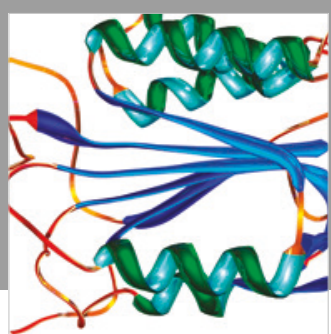

Disease Markers
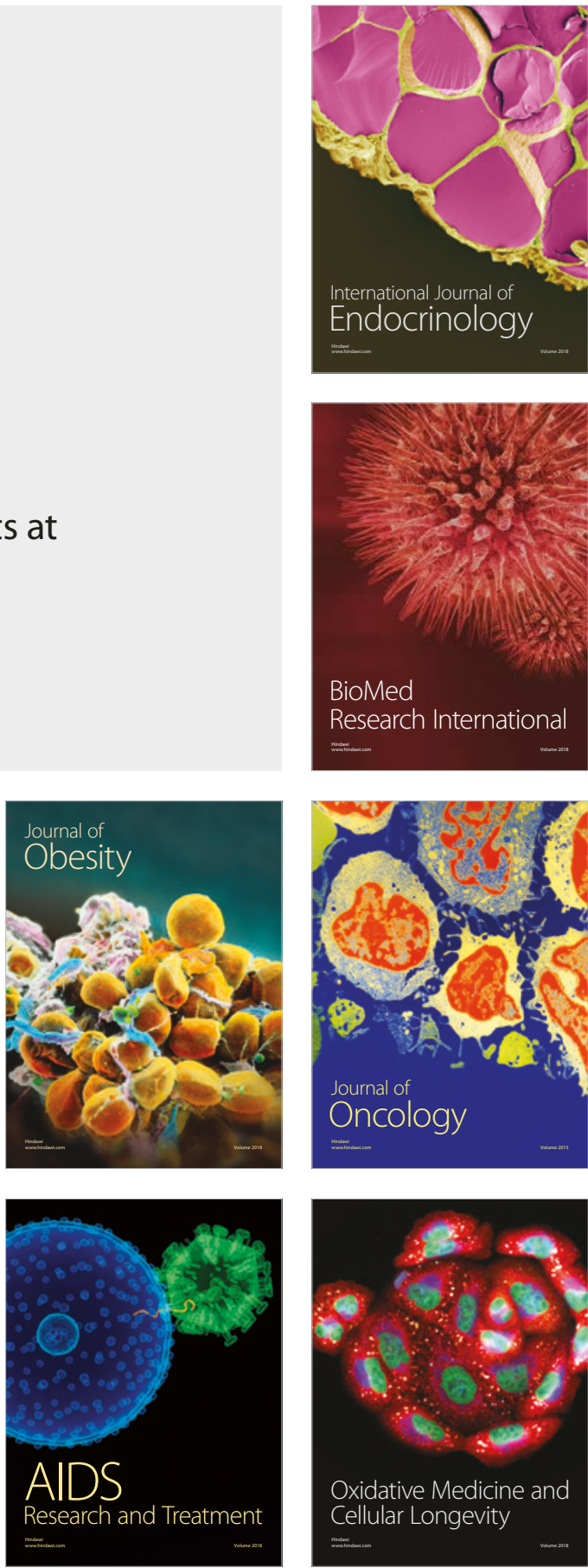